\title{
SUMMARY
}

Considerable amount of works has been reported on the study of cerebral circulation, but not so many reports are available on the subject of stem circulation.

The authers performed experimental studies on the hemodynamics of the Galen's vein of dogs with acute closed head injury, and the results are reported in this paper.

\section{Serial Observations of Eye Grounds in Patients of Head Injury}

\author{
Shigeo Watanabe and *Yoshiro Takeuchi \\ Chukyo General Hospital
}

We have attempted to investigate a change of artery and vein of eye ground, in order to determine a clinical relation between patient's condition and findings of retinal vessels, using Portable Fundus Camera for head injury.

1) Contraction of artery and dilatation of vein in retina were observed in unconscious patients at acute stage of head injury. For such patients, fluid transfusion (polyvinylpyrrolidone, etc.) of $100-300$ c.c. in volume were useful and important procedure which improved the retinal circulation and then clinical improvements were obtained.

2) Clinical improvement ran parallel with circulatory betterment in retina.

3) In case of acute intracranial hematoma, dimness around papilla was observed together with circulatory disturbance in retina, such a change of which advanced to choked disk.

4) There were observed significant changes in retina of post-traumatic syndrome cases.

\section{Surgical Treatment of the Optic Nerve Injury}

Y. Inaba, K. Ishizuka, N. Hoshiko, M. Yanagisawa, T. Nonaka,

M. Fuse, Y. Uchida, T. MiYanaga, A. Yamagami,

K. Ohara and H. Maruta

Dept of Surgery, Tokyo Medicodental College 